\title{
Ultrafast dynamics of carriers and phonons of photoinjected double-plasma in aluminium nitride
}

\author{
C. Gonçalves Rodrigues ${ }^{a}$ and R. Luzzi ${ }^{b}$ \\ ${ }^{a}$ School of Exact Sciences and Computing, Pontifical Catholic University of Goiás, \\ C.P. 86, 74605-010 Goiânia, Goiás, Brazil. \\ e-mail: cloves@pucgoias.edu.br \\ ${ }^{b}$ Condensed Matter Physics Department, Institute of Physics "Gleb Wataghin" \\ State University of Campinas-Unicamp, 13083-859 Campinas, S.P., Brazil.
}

Received 7 December 2020; accepted 22 December 2020

\begin{abstract}
Aluminum nitride is attracting great interest of the industry and scientific community due to its interesting properties. In this paper is performed a theoretical study on the ultrafast transient transport properties of photoinjected carriers in wurtzite AlN subjected to electric fields up to $80 \mathrm{kV} / \mathrm{cm}$. For this, the Nonequilibrium Statistical Operator Method was used. The evolution towards the steady state of drift velocity of carriers (electrons and holes) and nonequilibrium temperature (carriers and phonons) subpicosecond scale were determined.
\end{abstract}

Keywords: Aluminium nitride; plasma semiconductor; photoinjected semiconductor.

PACS: 81.05.Ea; 63.20.kd; 78.47.+p; 72.20.Ht; 05.70.Ln

DOI: https://doi.org/10.31349/RevMexFis.67.318

\section{Introduction}

The wide-band gap semiconductor materials such as silicon carbide, gallium nitride, aluminum nitride, diamond, and others, are often referred to as third-generation semiconductor materials. Aluminum nitride (AIN) with a large direct band gap of $6.2 \mathrm{eV}$ is a typical representative of these materials. Due to its ultra-wide direct band gap, high carrier mobility, large breakdown field, high thermal conductivity, high volume resistivity, high chemical and thermal stabilities, high dielectric strength, and low dielectric loss, make AIN an excellent material for novel electronic and opto-electronic device applications: $i$ ) laser diodes (LDs), deep-ultraviolet (DUV) solid-state light-emitting diodes (LEDs); ii) high frequency, high-power, and high-temperature electronic applications; iii) for packaging and substrates for high-density or high-power assemblies of microelectronic components [123].

The study of photoinjected (or photoexcited) carriers in AlN-based semiconductors can provide information regarding both their phonon and carrier dynamics properties, which are important for a better device design. Basic research regarding the ultrafast transport transient of hot carriers is relevant for technological applications in sub-micron devices.

In this paper, an analysis was performed to investigate the ultrafast transport properties of photoinjected carriers in wurtzite AlN subjected to electric fields up to $80 \mathrm{kV} / \mathrm{cm}$. The optical and transport properties of semiconductors have been studied mainly by using Nonequilibrium Green's Functions Techniques [24], Monte Carlo simulation [25,26], balance equation approach [27,28], Boltzmann transport equations $[26,29,30]$, etc. In this paper was used the "Nonequilibrium Statistical Operator Method" (NESOM) [31-35]. The NE-
SOM is a powerful formalism that seems to offer an elegant and concise way for an analytical treatment in the theory of irreversible processes, adequate to deal with a large class of experimental situations, and physically clear picture of irreversible processes. The NESOM is also practical and efficient in the study of the optical and carrier dynamics in semiconductors [36-42]. More specifically, a Non-Equilibrium Quantum Kinetic Theory [43] derived from NESOM was used in this paper.

\section{The evolution equations}

We consider a pure WZ AlN sample, with a concentration $n$ of electron-hole pairs created by an intense laser light pulse. The photoinjected carriers were far from equilibrium with the lattice but thermalized between themselves by Coulomb interaction after a fraction of picosecond. A constant electric field $\mathcal{E}$ was applied to the sample to accelerate the carriers as well as relax the energy and momentum to the phonon field simultaneously. The sample was in contact with a thermal reservoir at temperature $T_{0}$.

The following variables were used to describe the nonequilibrium thermodynamic state of the system: $i$ ) The reciprocal of the carrier nonequilibrium temperature, $\beta_{c}^{*}(t)=$ $\left(1 / k_{B} T_{c}^{*}\right)$; ii) The reciprocal of the longitudinal optical phonon nonequilibrium temperature, $\beta_{L O}^{*}(t)=1 /\left(k_{B} T_{L O}^{*}\right)$; iii) The reciprocal of the acoustic phonon nonequilibrium temperature, $\left.\beta_{A C}^{*}(t)=1 /\left(k_{B} T_{A C}^{*}\right) ; i v\right)$ The variables, $-\beta_{c}^{*}(t) \mu_{e}^{*}(t)$ and $-\beta_{c}^{*}(t) \mu_{h}^{*}(t)$, where $\mu_{e}^{*}(t)$ and $\mu_{h}^{*}(t)$ are the electron and hole chemical potentials, respectively; $v$ ) The variables: $-\beta_{c}^{*}(t) \mathbf{v}_{e}$ and $-\beta_{c}^{*}(t) \mathbf{v}_{h}$, where $\mathbf{v}_{e}$ and $\mathbf{v}_{h}$ are the electron and hole drift velocities, respectively. Here, $k_{B}$ is the Boltzmann constant. Note that the term "nonequi- 
librium temperature", symbolized by $T^{*}$, is also referred to as "quasi-temperature".

The nonlinear coupled set of integro-differential generalized transport equations that govern the time-evolution of these nonequilibrium thermodynamic variables is based on nonlinear quantum kinetic theory, which is based on a nonequilibrium statistical ensemble method [43], and the Markovian approximation is used [44]. The equations of evolution for the carriers' linear momentum, carriers' energy and LO, TO, AC phonons energy are as follows [43]:

$$
\begin{aligned}
\frac{d}{d t} \mathbf{P}_{e}(t) & =J_{\mathbf{P}_{e}}^{(1)}+J_{\mathbf{P}_{e}}^{(2)}(t), \\
\frac{d}{d t} \mathbf{P}_{h}(t) & =J_{\mathbf{P}_{h}}^{(1)}-J_{\mathbf{P}_{h}}^{(2)}(t), \\
\frac{d}{d t} E_{c}(t) & =J_{E_{c}}^{(1)}(t)-J_{E_{c}}^{(2)}(t), \\
\frac{d}{d t} E_{L O}(t) & =J_{E_{L O}}^{(2)}(t)-J_{L O, a n}^{(2)}(t), \\
\frac{d}{d t} E_{A C}(t) & =J_{E_{A C}}^{(2)}(t)+J_{L O, a n}^{(2)}(t)-J_{E_{A C}, d i f}^{(2)}(t) .
\end{aligned}
$$

In Eqs. (1) and (2) the first term on the right side is the force produced by the electric field $\left(J_{\mathbf{P}_{e}}^{(1)}=-N e \mathcal{E}\right.$, $\left.J_{\mathbf{P}_{h}}^{(1)}=N e \mathcal{E}\right)$, where $N=n V$, with $n$ being the concentration of electron-hole pairs, and $V$ the volume of the sample. In Eqs. (1) and (2) the second term on the right side is the rate of momentum transferred to the phonons [43].

In Eq. (3) the first term on the right side accounts for the rate of energy pumped to the carrier system owing to the the electric field,

$$
J_{E_{c}}^{(1)}(t)=e \mathcal{E} \cdot\left(\frac{\mathbf{P}_{h}(t)}{m_{h}^{*}}-\frac{\mathbf{P}_{e}(t)}{m_{e}^{*}}\right),
$$

and the second term on the right side represents the rate of excess energy transferred to the phonons [43].

The populations of the LO- and AC- phonons [43] are expressed as follows:

$$
\begin{aligned}
& \nu_{L O}(t)=\frac{1}{e^{\beta_{L O}^{*}(t) \hbar \omega_{L O}}-1}, \\
& \nu_{A C}(t)=\frac{1}{e^{\beta_{A C}^{*}(t) \hbar \omega_{A C}}-1},
\end{aligned}
$$

where $\omega_{(L O)}$ and $\omega_{(A C)}$ are the frequencies of the (LO) or (AC) phonons, respectively. We take the "Einstein model" dispersionless frequency relation for LO phonons, that is: $\hbar \omega_{\mathbf{q}, \mathrm{LO}}=\hbar \omega_{0}$ (i.e. all LO phonons have the same angular frequency $\omega_{0}$ ), and the "Debye model" dispersionless frequency relation for $\mathrm{AC}$ phonons, that is: $\hbar \omega_{\mathbf{q}, \mathrm{AC}}=\hbar q v_{s}$, where $v_{s}$ is the sound velocity. Note that the AC and LO phonons were considered to be internally thermalized, disregarding possible differentiated distribution of populations in reciprocal space as a result of what can be termed as FröhlichCherenkov effect [45-48]. The inhomogeneous distribution in reciprocal space is restricted to a very small region of the
Brillouin zone [45-48] and such effect can be neglected in the present study.

In Eqs. (4) and (5) the first term on the right side are the gain of energy pumped on the phonons (LO and AC, respectively) by the "hot" carriers, and the second term on the right side in Eqs. (4) and (5) are the transfer, via anharmonic effects, of the energy of LO-phonons to the AC-phonons [43]

$$
J_{L O, a n}^{(2)}(t)=\frac{V \hbar \omega_{L O}}{V_{\text {cell }}} \frac{\left[\nu_{L O}(t)-\nu_{A C}(t)\right]}{\tau_{o p, a n}},
$$

where $\tau_{o p, a n}$ is the relaxation time obtained from the band width from Raman scattering experiments [49] and $V_{\text {cell }}$ is the volume of the unit cell.

The second term on the right side in Eq. (5) is the same of Eq. (4), but with the opposite sign. The last term in Eq. (5) is the contribution of thermal diffusion to the reservoir (thermal bath) at temperature $T_{0}$.

Finally, it noteworthy that, to close the system of equations, we must express the linear momentum of the carriers, and the energies of the phonons and carriers, as follows [43]:

$$
\begin{aligned}
E_{c} & =3 k_{B} T_{c}^{*}(t)+N \frac{m_{e}^{*} v_{e}^{2}(t)}{2}+N \frac{m_{h}^{*} v_{h}^{2}(t)}{2}, \\
\mathbf{P}_{e}(t) & =N m_{e}^{*} \mathbf{v}_{e}(t), \\
\mathbf{P}_{h}(t) & =N m_{h}^{*} \mathbf{v}_{h}(t), \\
E_{L O}(t) & =\frac{V \hbar \omega_{L O}}{V_{\text {cell }}} \nu_{L O}(t) . \\
E_{A C}(t) & =\frac{3 V}{V_{\text {cell }}} \frac{1}{\beta_{A C}^{*}(t)} .
\end{aligned}
$$

\section{Results}

The calculations were performed in WZ AlN for photoinjected carriers with a concentration $n=10^{18} \mathrm{~cm}^{-3}$ and an excess energy of $0.6 \mathrm{eV}$ per pair gained from photon absorption. After Coulomb thermalization, the initial carrier tempe-

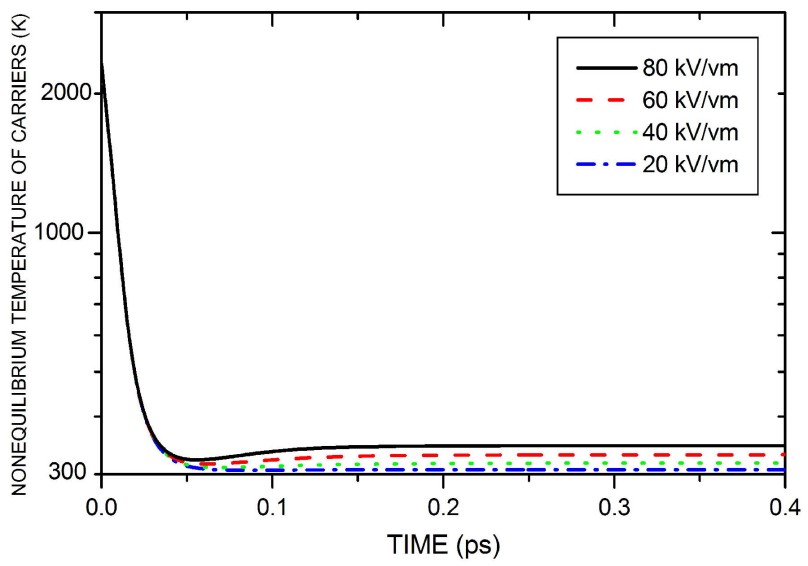

FIGURE 1. Time evolution of carriers nonequilibrium temperature in photoinjected WZ AlN for four electric field intensities. 


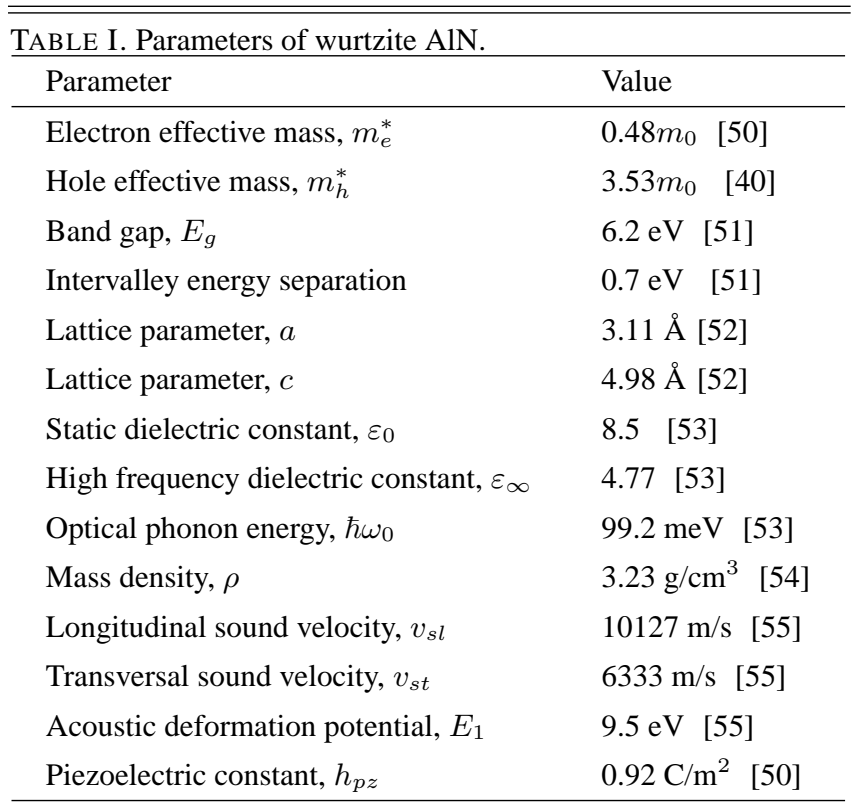

rature was approximately $T_{c}(0)=2321 \mathrm{~K}$. The thermal bath temperature considered was $T_{0}=300 \mathrm{~K}$, and the carrier scattering mechanisms considered in the numerical calculations included piezoelectric, deformation acoustic and polar optical (or Fröhlich's interaction). Table I summarizes the material parameters of the WZ AIN used in numerical calculations in this paper. In Table I, $m_{0}$ is the free electron mass.

Figure 1 shows, for four values of the electric field intensities, the time evolution of the nonequilibrium temperature of the carriers, describing a extremely rapid process of relaxation of energy to the lattice. It can be noticed that for $\mathcal{E} \lesssim$ $80 \mathrm{kV} / \mathrm{cm}$ the carriers cool down to a steady state in less than $0.4 \mathrm{ps}$.

Figure 2 shows, for an electric field strength of $80 \mathrm{kV} / \mathrm{cm}$, the evolution of the rate of change of the carriers nonequilibrium temperature with respect to time, $d T_{c}^{*} / d t$. The rate of change (in arbitrary units) is negative for up to $0.05 \mathrm{ps}$. The arrow in Fig. 2 indicates this point. For values greater than

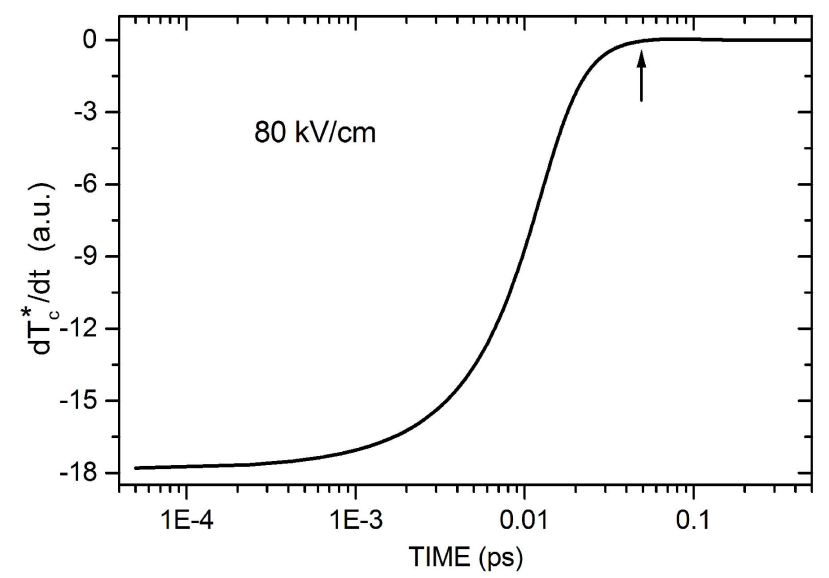

FIGURE 2. Time evolution of rate of change of carriers nonequilibrium temperature in photoinjected $\mathrm{WZ}$ AlN, in arbitrary units, for an electric field of $80 \mathrm{kV} / \mathrm{cm}$.
$0.05 \mathrm{ps}$ the rate of change becomes positive. The behavior of the curves for the other electric field values are similar. Note that Eq. (3) comprises two terms: $J_{E_{c}}^{(1)}$ and $J_{E_{c}}^{(2)}$. The first term, $J_{E_{c}}^{(1)}$, is a source term. It is the energy received of the carriers due to applied electric field. The second term, $J_{E_{c}}^{(2)}$, is the dissipation of energy: the carriers lose his initial excess of energy (gained in photon absorption processes) for the phonons. In larger electric fields $J_{E_{C}}^{(1)}$ is also larger, and the total dissipation of energy $\left(J_{E_{c}}^{(1)}+J_{E_{c}}^{(2)}\right)$ is slower that for smaller values of electric fields.

Figure 3 shows the time evolution of the electron (Fig. 3a) and hole (Fig. 3b) drift velocities for four electric field intensities. After a transient regime in the order of picoseconds, a steady state was achieved. Similar to Fig. 1, as larger the electric field more time the system takes to reach the steady state. During the transient regime, it can be noticed the behavior of the maxima existence for holes, with one of the maxima corresponding to a velocity drift overshoot for fields larger than approximately $40 \mathrm{kV} / \mathrm{cm}$. The structured ultrafast hole drift velocity in photoinjected WZ AIN is explained in this paper uniquely through the crossover of the evolution curves for the transport and momentum relaxation times, whose definition is based on the nonequilibrium variables used to describe the system [56]. The values obtained for the hole drift velocities
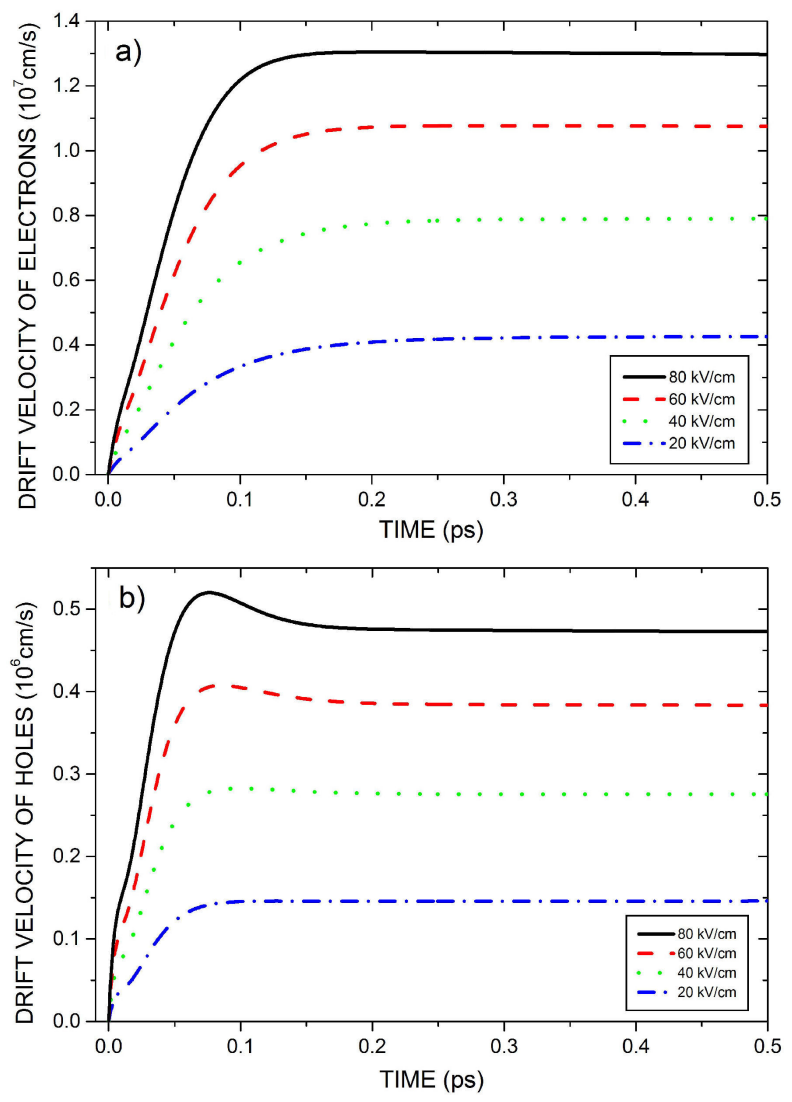

FIGURE 3. Time evolution of drift velocity: a) electrons and b) holes, in photoinjected WZ AlN for four electric field intensities. 

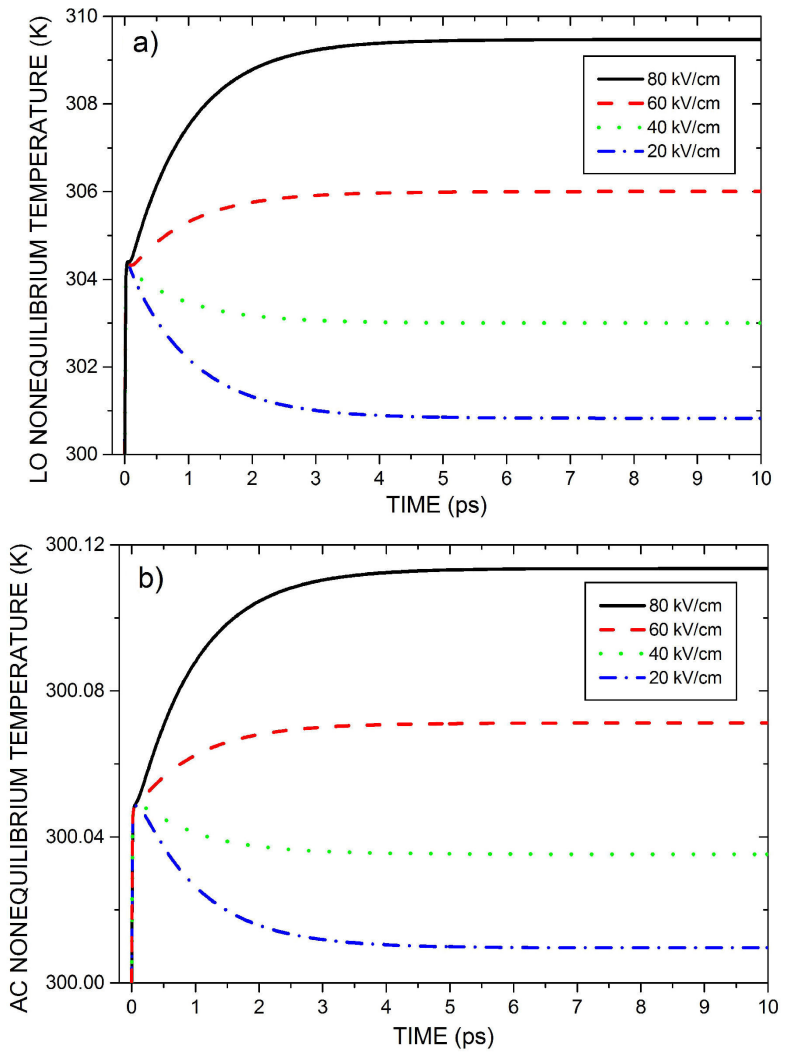

FIGURE 4. Time evolution of nonequilibrium temperature of LOphonons (top) and AC-phonons (bottom) in photoinjected WZ AlN for four electric field intensities.

are much smaller than those of electrons. This fact can be ascribed to the holes having a higher effective mass than the electrons: $m_{h}^{*} / m_{e}^{*} \simeq 7.3$ (see Table I).

Using the results of Fig. 3, we can derive the mobility, $\mathcal{M}_{e(h)}$, of the electrons and holes, as given by $\mathcal{M}_{e(h)}=$ $\left|v_{e(h)}\right| /|\mathcal{E}|$. For a electric field of $20 \mathrm{kV} / \mathrm{cm}$ the electron and hole mobility are approximately $\mathcal{M}_{e} \simeq 213 \mathrm{~cm}^{2} / \mathrm{V} \cdot \mathrm{s}$ and $\mathcal{M}_{h} \simeq 21.3 \mathrm{~cm}^{2} / \mathrm{V} \cdot \mathrm{s}$, respectively. These values are among the range of theoretical and experimental data obtained for the WZ AlN [15,50,53,57].

Figure 4a) displays the time evolution of the nonequilibrium temperature of the LO-phonons for four different values of the electric field intensities. We can verify that the LO-phonon quasitemperature does not increase appreciably above equilibrium temperature $T_{0}$ : approximately $3 \%$ for an electric field of $80 \mathrm{kV} / \mathrm{cm}$, the higher field we have considered. Figure $4 b$ ) shows the time evolution of the nonequilibrium temperature of the AC-phonons for four different values of the electric field intensities. It is possible to verify that the temperature increase of the AC-phonons are irrelevant.

\section{Conclusions}

In this study, we obtain equations that govern the time evolution of the nonequilibrium state of highly photoinjected double plasma in the semiconductor WZ AlN subjected to electric field intensities to $80 \mathrm{kV} / \mathrm{cm}$. For this study, we used a nonlinear quantum transport theory derived from the Nonequilibrium Statistical Operator Method [31-35]. We presented a study regarding ultrafast transport transient characteristics, focusing on the dependency of the nonequilibrium temperatures (carriers and phonons) as well as the electron and hole drift velocities on the electric field strength. Maxima of the hole drift velocity in its transient regime should be observable depending on the evolution of the nonequilibrium macroscopic state of the photoinjected carriers in WZ AIN. The transient regime occurred on a picosecond scale. The longitudinal optical phonons are slightly heated up in scattering events involving Fröhlich interaction with the carriers, and the temperature increase of the AC-phonons was irrelevant.
1. Y.G. Cao et al., Blue emission and Raman scattering spectrum from AlN nanocrystalline powders, J. Cryst. Growth 213 (2000) 198. https://doi.org/10.1016/ S0022-0248(00)00379-1

2. S.C. Jain, M. Willander, J. Narayan, and R. Overstraeten, IIInitrides: Growth, characterization, and properties, J. Appl. Phys. 87 (2000) 965. https://doi.org/10.1063/1. 371971

3. M.E. Levinshtein, S.L. Rumyantsev, and M.S. Shur, Properties of Advanced Semiconductor Materials: GaN, AlN, InN, BN, $\mathrm{SiC}$, SiGe (Wiley, New York, 2001).

4. A.G. Bhuiyan, A. Hashimoto, and A. Yamamoto, Indium nitride ( $\mathrm{InN})$ : A review on growth, characterization, and properties, J. Appl. Phys. 94 (2003) 2779. https://doi.org/ $10.1063 / 1.1595135$

5. M.S. Shur and R.F. Davis, GaN-Based Materials and De- vices: Growth, Fabrication, Characterization and Performance (World Scientific, River Edge, 2004).

6. M. Iwata, K. Adachi, S. Furukawa, and T. Amakawa, Synthesis of purified AlN nano powder by transferred type arc plasma, $J$. Phys. D Appl. Phys. 37 (2004) 1041. https://doi.org/ 10.1088/0022-3727/37/7/014

7. Y. Taniyasu, M. Kasu, and T. Makimoto, An aluminium nitride light-emitting diode with a wavelength of 210 nanometres, Nature 441 (2006) 325. https://doi.org/10.1038/ nature04760

8. Y. Taniyasu and M. Kasu, Aluminum nitride deep-ultraviolet light-emitting p-n junction diodes, Diam. Relat. Mater. 17 (2008) 1273. https://doi.org/10.1016/j. diamond.2008.02.042

9. J. Wu, When group-III nitrides go infrared: New properties and perspectives, J. Appl. Phys. 106 (2009) 011101. https: //doi.org/10.1063/1.3155798 
10. S.J. Pearton, C.R. Abernathy, and F. Ren, Gallium Nitride Processing for Electronics, Sensors and Spintronics (Springer, New York, 2010).

11. N.S. Kanhe et al., Understanding the growth of micro and nano-crystalline AlN by thermal plasma process, J. Cryst. Growth 339 (2012) 36. https: / / doi .org/10.1016/j . jcrysgro.2011.11.011

12. R.R. Sumathi, Bulk AlN single crystal growth on foreign substrate and preparation of free-standing native seeds, Cryst. Eng. Comm. 15 (2013) 2232. https://doi.org/10.1039/ C2CE26599K

13. E.A. Jones, F. Wang, and D. Costinett, Review of commercial GaN power devices and GaN-based converter design challenges, IEEE J. Emerg. Sel. Topics Power Electron. 4 (2016) 707. https://doi.org/10.1109/JESTPE. 2016.2582685

14. G. Meneghesso et al., Reliability and parasitic issues in GaN-based power HEMTs: a review, Semicond. Sci. Technol. 31 (2016) 093004. https://doi.org/10.1088/ 0268-1242/31/9/093004

15. H. Wu and R. Zheng, Single Crystal AlN: Growth by Modified Physical Vapor Transport and Properties, In: III-Nitride Materials, Devices and Nano-Structures, edited By: Zhe Chuan Feng (World Scientific Publishing, Singapore, 2017), pp. 151. https://doi.org/10.1142/9781786343192_0005

16. J. Zagoraca, D. Zagoraca, D. Jovanović, J. Luković, and B. Matović, Ab initio investigations of structural, electronic and mechanical properties of aluminum nitride at standard and elevated pressures, J. Phys. Chem. Solid. 122 (2018) 94. https: //doi.org/10.1016/j.jpcs.2018.06.020

17. D.M. Spiridonov et al., Spectrally resolved thermoluminescence in electron irradiated AlN submicrocrystals, Radiation Measurements 122 (2019) 91. https: / / doi.org/10. $1016 / j . r a d m e a s .2019 .02 .001$

18. K. Teker, Dielectrophoretic Assembly of Aluminum Nitride (AIN) Single Nanowire Deep Ultraviolet Photodetector, J. Nano Research 60 (2019) 86. https://doi.org/10. 4028/www.scientific.net/JNanoR.60.86

19. H. Wei et al., Interface modification for high-efficient quantum dot sensitized solar cells using ultrathin aluminum nitride coating, Appl. Surf. Sci. 476 (2019) 608. https://doi.org/ $10.1016 / j$.apsusc.2019.01.144

20. Z. Ren et al., III-nitride Deep UV LED without Electron Blocking Layer, IEEE Photonics Journal 11 (2019) 8200511. https://doi.org/10.1109/JPHOT.2019. 2902125

21. N. Alfaraj et al., Deep-ultraviolet integrated photonic and optoelectronic devices: A prospect of the hybridization of group III-nitrides, III-oxides, and two-dimensional materials, J. Semicond. 40 (2019) 121801. https://doi.org/10.1088/ $1674-4926 / 40 / 12 / 121801$

22. R. Lin et al., X-ray radiation excited ultralong $(>20,000$ seconds) intrinsic phosphorescence in aluminum nitride single-crystal scintillators, Nature Communications 11 (2020) 4351. https://doi.org/10.1038/ s41467-020-18221-1
23. B. Choudhuri and A. Mondal, Group III-Nitrides and Other Semiconductors for Terahertz Detector. In: Emerging Trends in Terahertz Solid-State Physics and Devices, edited by A. Biswas, A. Banerjee, A. Acharyya, H. Inokawa and J. Roy (Springer, Singapore, 2020). http://doi-org-443.webvpn.fjmu.edu.cn/ $10.1007 / 978-981-15-3235-1 \_12$

24. D.Y. Xing and C.S. Ting, Green's-function approach to transient hot-electron transport in semiconductors under a uniform electric field, Phys. Rev. B 35 (1987) 3971. https://doi. org/10.1103/PhysRevB.35.3971

25. N. Ma et al., Hole mobility in wurtzite InN, Appl. Phys. Lett. 98 (2011) 192114. https: / /doi.org/10.1063/1. 3592257

26. P. Siddiqua and S.K. O'Leary, Electron transport within the wurtzite and zinc-blende phases of gallium nitride and indium nitride, J. Mater. Sci.: Mater. Electron. 29 (2018) 3511. https://doi.org/10.1007/s10854-017-8324-1

27. L.F. Mao, Quantum coupling and electrothermal effects on electron transport in high-electron mobility transistors, Pramana - J. Phys. 93 (2019) 11. https://doi.org/10. 1007/s12043-019-1769-4

28. T. Linn, K. Bittner, H.G. Brachtendorf, and C. Jungemann, Simulation of THz oscillations in semiconductor devices based on balance equations, J. Sci. Comput. 85 (2020) 6. https: //doi.org/10.1007/s10915-020-01311-z

29. V. Stefano, An energy transport model describing electrothermal transport in silicon carbide semiconductors, J. Comput. Theor. Transport 46 (2017) 379. https://doi.org/10. $1080 / 23324309.2017 .1352513$

30. S.M. Hong and J.H. Jang, Transient simulation of semiconductor devices using a deterministic Boltzmann equation solver, IEEE J. Electron Devices Soc. 6 (2018) 156. https: / / doi. org/10.1109/JEDS.2017.2780837

31. D.N. Zubarev, Nonequilibrium Statistical Thermodynamics (Consultants Bureau, New York, 1974).

32. D.N. Zubarev, V. Morozov, and G. Röpke, Statistical Mechanics of Nonequilibrium Processes, Vols. 1 and 2 (Akademie Verlag Wiley VCH Publishing Group, Berlin, 1996 and 1997).

33. R. Luzzi, A.R. Vasconcellos, and J.G. Ramos, Predictive Statistical Mechanics: a Nonequilibrium Statististical Ensemble Formalism (Kluwer Academics, Dordrecht, The Netherlands, 2002).

34. R. Luzzi, A.R. Vasconcellos, and J.G. Ramos, Statistical Foundations of Irreversible Thermodynamics (Teubner-Bertelsmann Springer, Stuttgart, 2000).

35. R. Luzzi, A.R. Vasconcellos, J.G. Ramos, and C.G. Rodrigues, Statistical irreversible thermodynamics in the framework of Zubarev's nonequilibrium statistical operator method, Theor. Math. Phys. 194 (2018) 4. https: //doi.org/10.1134/ S0040577918010038

36. C.G. Rodrigues, A.R. Vasconcellos, and R. Luzzi, Optical properties of III-nitrides in electric fields, Eur. Phys. J. B. 72 (2009) 67. https://doi.org/10.1140/epjb/ e2009-00332-y 
37. C.G. Rodrigues, A.R. Vasconcellos, R. Luzzi, and V.N. Freire, Urbach's tail in III-nitrides under an electric field, J. Appl. Phys. 90 (2001) 1879. https://doi.org/10.1063/1. 1384859

38. C.G. Rodrigues, A.R. Vasconcellos, and R. Luzzi, NonLinear electron mobility in n-doped III-Nitrides, Braz. J. Phys. 36 (2006) 255. http://www.sbfisica.org.br/bjp/ files/v36_255.pdf

39. C.G. Rodrigues, C.A.B. Silva, A.R. Vasconcellos, J.G, Ramos, and R. Luzzi, The role of nonequilibrium thermo-mechanical statistics in modern technologies and industrial processes: an overview, Braz. J. Phys. 40 (2010) 63. http://www. sbfisica.org.br/bjp/files/v40_63.pdf

40. C.G. Rodrigues, A.R. Vasconcellos, and R. Luzzi, Nonlinear hole transport and nonequilibrium thermodynamics in group III-nitrides under the influence of electric fields, J. Appl. Phys. 102 (2007) 073714. https: //doi.org/10.1063/ 1.2785976

41. C.G. Rodrigues, Electron mobility in n-doped zinc sulphide, Microelectron. J. 37 (2006) 657. https://doi.org/10. $1016 / j . m e j o .2005 .05 .015$

42. C.G. Rodrigues, V.N. Freire, A.R. Vasconcellos, and R. Luzzi, Electron mobility in nitride materials, Braz. J. Phys. 32 (2002) 439. http://www.sbfisica.org.br/bjp/ files/v32_439.pdf

43. C.G. Rodrigues, A.R. Vasconcellos, and R. Luzzi, A kinetic theory for nonlinear quantum transport, Transp. Theory Stat. Phys. 29 (2000) 733. http://dx.doi.org/10.1080/ 00411450008200000

44. L. Lauck, A.R. Vasconcellos, and R. Luzzi, A nonlinear quantum transport theory, Physica A 168 (1990) 789. https: //doi.org/10.1016/0378-4371(90)90031-M

45. C.G. Rodrigues, A.R. Vasconcellos, and R. Luzzi, Nonlinear transport in n-III-nitrides: Selective amplification and emission of coherent LO phonons, Solid State Commun. 140 (2006) 135. https://doi.org/10.1016/j.ssc.2006.08.015

46. C.G. Rodrigues, A.R. Vasconcellos, and R. Luzzi, Evolution kinetics of nonequilibrium longitudinal-optical phonons generated by drifting electrons in III-nitrides: longitudinal-opticalphonon resonance, J. Appl. Phys. 108 (2010) 033716. https : //doi.org/10.1063/1.3462501

47. C.G. Rodrigues, A.R. Vasconcellos, and R. Luzzi, Drifting electron excitation of acoustic phonons: Cerenkov-like effect in n-GaN, J. Appl. Phys. 113 (2013) 113701. https: / / doi. org/10.1063/1.4795271

48. C.G. Rodrigues, F.S. Vannucchi, and R. Luzzi, Nonequilibrium Bose-Einstein-like condensation, Advanced Quantum Technologies 1 (2018) 201800023. https://doi. org/10.1002/qute.201800023
49. R.K. Chang, J.M. Ralston, and D.E. Keating, Ligth Scattering Spectra of Solids I, edited by G.B. Wright (Springer, New York, 1969), pp. 369

50. P. Siddiqua, W.A. Hadi, M.S. Shur, and S.K. O'Leary, A 2015 perspective on the nature of the steady-state and transient electron transport within the wurtzite phases of gallium nitride, aluminum nitride, indium nitride, and zinc oxide: a critical and retrospective review, J. Mater. Sci.: Mater. Electron 26 (2015) 4475 .

51. S. Wang et al., Comparison the electron momentum and energy relaxation process in wurtzite $\mathrm{GaN}, \mathrm{InN}$ and $\mathrm{AlN}$ by Monte Carlo method, Solid State Commun. 288 (2019) 68. https: //doi.org/10.1016/j.ssc.2018.11.018

52. H. Wu and R. Zheng, Single Crystal AlN: Growth by Modified Physical Vapor Transport and Properties, In: III-Nitride Materials, Devices and Nano-Structures, edited By: Z. C. Feng (World Scientific Publishing, Singapore, 2017), pp. 151. https://doi.org/10.1142/9781786343192_0005

53. S.K. O'Leary, P. Siddiqua, W.A. Hadi, B.E. Foutz, M.S. Shur, and L.F. Eastman, Electron Transport Within III-V Nitride Semiconductors, In: Springer Handbook of Electronic and Photonic Materials (Part D: Materials for Optoelectronics and Photonics), edited by S. Kasap and P. Capper (Springer International Publishing, Berlin, 2017). https://doi.org/10. 1007/978-3-319-48933-9_32

54. S.K. O'Leary, B.E. Foutz, M.S. Shur, and L.F. Eastman, Steady-State and Transient Electron Transport Within the IIIV Nitride Semiconductors, GaN, AlN, and InN: A Review, J. Mater. Sci.: Mater. Electron. 17 (2006) 87. https ://doi. org/10.1007/s10854-006-5624-2

55. S. Contreras et al., High temperature electrical transport study of Si-doped AlN, Superlattice. Microst. 98 (2016) 253. https://doi.org/10.1016/j.spmi.2016.08. 038

56. C.G. Rodrigues, A.R. Vasconcellos, R. Luzzi, and V.N. Freire, Transient transport in III-nitrides: interplay of momentum and energy relaxation times, J. Phys-Condens. Mat. 19 (2007) 346214. https://doi.org/10.1088/ 0953-8984/19/34/346214

57. H. Arabshahi, M. Izadifard, and A. Karimi, Calculation of elecron mobility in WZ-AlN and ZB-AlN at low electric field, International Journal of Science, Environment and Technology 1 (2012) 395. https://doi .org/10 .15580/GJSETR . 2012.3 .10031275

58. http://www.ioffe.ru/SVA/NSM/Semicond/AlN/ hall.html 